\title{
The Exponential Sampling Theorem of Signal Analysis and the Reproducing Kernel Formula in the Mellin Transform Setting
}

\author{
Carlo Bardaro \\ Department of Mathematics and Computer Science, University of Perugia, Via Vanvitelli 1 \\ Perugia, 06123, Italy \\ carlo.bardaro@unipg.it \\ Paul Leo Butzer \\ Lehrstuhl A fuer Mathematik, RWTH Aachen, Templergraben 55 \\ Aachen, D-52056, Germany \\ butzer@rwth-aachen.de \\ Ilaria Mantellini \\ Department of Mathematics and Computer Sciences, University of Perugia, Via Vanvitelli 1 \\ Perugia, 06123, Italy \\ mantell@dmi.unipg.it
}

\begin{abstract}
The Shannon sampling theory of signal analysis, the so-called WKSsampling theorem, which can be established by methods of Fourier analysis, plays an essential role in many fields. The aim of this paper is to study the so-called exponential sampling theorem (ESF) of optical physics and engineering in which the samples are not equally spaced apart as in the Shannon case but exponentially spaced, using the Mellin transform methods. One chief aim of this paper is to study the reproducing kernel formula, not in its Fourier transform setting, but in that of Mellin, for Mellin-bandlimited functions as well as an approximate version for a less restrictive class, the MRKF, namely for functions which are only approximately Mellin-bandlimited. The rate of approximation for such signals will be measured in terms of the strong Mellin fractional differential operators recently studied by the authors. The final aim is to show that the exponential sampling theorem ESF is equivalent to the MRKF for Mellinbandlimited functions (signals) in the sense that each is a corollary of the other. Three graphical examples are given, as illustration of the theory.
\end{abstract}

Key words and phrases: Mellin transform, Mellin band-limited functions, Mellin fractional derivative, exponential sampling, reproducing kernel formula

2010 AMS Mathematics Subject Classification - 30D10, 94A20, 42C15, $46 \mathrm{E} 22$ 


\section{Introduction}

One of the basic theorems of signal and Fourier analysis is the Shannon sampling theorem. It reads that if $f$ is bandlimited to an interval $[-\pi T, \pi T]$ for some $T>0$, i.e. the Fourier transform $\mathcal{F}[f](v):=(1 / \sqrt{2 \pi}) \int_{\mathbb{R}} f(u) e^{-i v u} d u$ vanishes outside $[-\pi T, \pi T]$, then $f$ can be completely reconstructed for all $x \in \mathbb{R}$ from its sampled value $f(k / T)$ taken at the nodes $k / T, k \in \mathbb{Z}$ equally spaced apart on $\mathbb{R}$, in terms of

$$
f(u)=\sum_{k=-\infty}^{+\infty} f\left(\frac{k}{T}\right) \operatorname{sinc}(T u-k), \quad(u \in \mathbb{R}),
$$

where $\operatorname{sinc}(u):=\sin (\pi u) / \pi u$. See e.g. A.I. Zayed [16], [25], J.R. Higgins [19].

Now in optical physics and engineering (see e.g. D. Casasent [17]) so called exponential sampling methods turned out to be of great importance from 1981 onwards. In this respect there are e.g. the papers by N. Ostrowsky et al. [20], M. Bertero and E. R. Pike [8] and F. Gori [18]. In fact, Bertero-Pike apply their results to problems of particle sizing in quasi-elastic light scattering (photon correlation spectroscopy) and small angle diffraction scattering (Frauenhofer diffraction). Here the samples are not equally spaced apart as in the Shannon case but exponentially spaced; such spacing is need in those applications where independent pieces of information accumulate near time $x=0$. In order to solve the problem involved these authors used - instead of Fourier transform methods as in the Shannon case - Mellin transform methods in a somewhat uncritical and formal way.

If $f$ is Mellin-bandlimited, thus for which the Mellin transform

$$
M[f](c+i t)=[f]_{M}^{\wedge}(c+i t):=\int_{0}^{+\infty} f(x) x^{c+i t-1} d x
$$

vanishes for all $|t|>T \pi$, some $c \in \mathbb{R}, T \in \mathbb{R}^{+}$, i.e. $f \in B_{c}^{\pi T}$, then the exponential sampling theorem (ESF) states that

$$
f(x)=\sum_{k=-\infty}^{+\infty} f\left(e^{k / T}\right) \operatorname{lin}_{c / T}\left(e^{-k} x^{T}\right), \quad\left(x \in \mathbb{R}^{+}\right),
$$

the role of the sinc function now being played by what is called the $\operatorname{lin}_{c}$ function, given by

$$
\operatorname{lin}_{c}(x):=x^{-c} \operatorname{sinc}(\log x)=\frac{x^{-c}}{2 \pi i} \frac{x^{\pi i}-x^{-\pi i}}{\log x}
$$

with $\operatorname{lin}_{c} 1=1$. Observe that the sampling points are now given by $1, e^{1 / T}, e^{2 / T}, \cdots$ lying to the right of 1 , and $\ldots e^{-100 / T}, e^{-99 / T}, \ldots, e^{-1 / T}$ lying between zero and 1. 
Until twenty years ago one would have studied the exponential sampling theorem by trying to reduce it to the Shannon sampling theorem by a change of function and of variable. Thus, writing ESF in terms of the function $F(x):=$ $f(\log x) x^{-c}$, then

$$
F(x)=f(\log x) x^{-c}=\sum_{k=-\infty}^{+\infty} f\left(\log \left(e^{k / T}\right)\right) e^{-k c / T} \operatorname{lin}_{c / T}\left(e^{-k} x^{T}\right) .
$$

But

$$
\operatorname{lin}_{c / T}\left(e^{-k} x^{T}\right)=\left(e^{-k} x^{T}\right)^{-c / T} \operatorname{sinc}\left(\log \left(e^{-k} x^{T}\right)\right)
$$

hence

$$
f(\log x)=\sum_{k=-\infty}^{\infty} f\left(\frac{k}{T}\right) \operatorname{sinc}(T \log x-k) .
$$

Under the change of variable $x=e^{u}$ there results the Shannon sampling formula (1). But as to the basic results of the present paper the situation will be shown to be quite different.

The main aim in the papers [13], [14] was to establish the exponential sampling theorem by a direct way, using the Mellin transform and its inversion theory. The first to use two formulae for the Mellin transform was Riemann in its famous memoir on the Riemann zeta function. This memoir gave rise to the study of the Mellin transform and its properties by many authors, including E. Cahen, O. Perron, R.H. Mellin, O.X. Schloemilch, G.H. Hardy and J.E. Littlewood, R.G. Mamedov ([21]). However, the proofs were mostly reduced to the corresponding ones for the Fourier (or even the bilateral Laplace) transform by a change of function and variables. On the other hand, although Fourier and Laplace transforms are also strictly related, both are neverthless treated as independent disciplines. Thanks again especially to number theorists, Mellin transform theory is gradually becoming an independent discipline. In their treatises on number theory, many now contain a whole chapter dealing with Mellin transform properties and their proofs (see e.g. [7], [22], [24]).

One of the authors, together with former students and colleagues, also proceeded in this fashion and examined in several articles the Mellin transform and its properties in a systematic form, under natural and minimal hypotheses upon the functions in question, from an elementary point of view, thus as an independent discipline (see [11], [12], [13], [14], [2], [15]).

By reducing the Mellin transform results by a change of variables and function to the Fourier ones, the hypotheses upon which the Mellin theory rests are often considered quite uncritically, certainly by no means in a unified, systematic fashion. Thus in the present paper these various aspects are taken seriously. In fact, one basis to the present paper is the authors paper [5] where it was shown that the very foundations of Mellin transform theory, studied there in a rigorous 
form, are quite different to those of Fourier transform theory, particularly since the operator of anti-differentiation, namely the integral $\int_{0}^{x} f(u) \frac{d u}{u}$ with derivative $\left(x \frac{d}{d x}\right) f(x)$, is fully different to the classical integral function and derivative. Application to various topics were also treated in [5], including fractional partial differential equations.

Section 2 is concerned with preliminary results and Section 3 recalls material concerning the strong Mellin fractional differential operator developed in [5], (see also [3], [4]), needed in evaluating later estimates. Section 4 is devoted to the exponential sampling formula (ESF) and the new Mellin reproduging kernel formula, both for Mellin bandlimited signals. In both cases their approximate versions are presented, namely those for not necessarily Mellin bandlimited signals; the errors involved are estimated in terms of the fractional differential operator.

The well-known classical reproducing kernel formula reads that if $f$ is bandlimited to $[-\pi T, \pi T]$, some $T>0$, then $f$ can be reproduced from itself via

$$
f(u)=T \int_{-\infty}^{+\infty} f(y) \operatorname{sinc} T(u-y) d y, \quad(u \in \mathbb{R}) .
$$

Its Mellin version, the new MRKF, which was announced in [4], will state that if $f \in X_{c}$ is Mellin bandlimited to $[-\pi T, \pi T]$, some $T \in \mathbb{R}^{+}$i.e., its Mellin transform $M[f](c+i t)=0$ for all $|t|>\pi T$ then

$$
f(x)=T \int_{0}^{+\infty} f(y) \operatorname{lin}_{c / T}\left(\left(\frac{x}{y}\right)^{T}\right) \frac{d y}{y} \quad\left(x \in \mathbb{R}^{+}\right) .
$$

Above

$$
X_{c}=\left\{f: \mathbb{R}^{+} \rightarrow \mathbb{C}: f(\cdot)(\cdot)^{c-1} \in L^{1}\left(\mathbb{R}^{+}\right)\right\} .
$$

Observe that the integral above, expressing the reproduction of the Mellinbandlimited $f \in X_{c}$ from itself in terms of "all" of its values, can be regarded as the integral analogue of the series (2) defining the ESF, which in turn is a "discrete" reproducing kernel formula.

Section 5 deals with the difficult question of the equivalence between ESF and MRKF, thus for Mellin bandlimited signals. Section 6 treats three examples together with their graphical representation, including the Mellin versions of Fejer and Abel-Poisson kernels. In both cases the truncation errors are considered and especially in the Mellin-Fejer case the partial sum of the sampling theorem is a good approximation.

\section{Basic Notions and Preliminary Results}

Let $C\left(\mathbb{R}^{+}\right)$be the space of all continuous functions defined on $\mathbb{R}^{+}$. For $1 \leq$ $p<+\infty$, let $L^{p}=L^{p}\left(\mathbb{R}^{+}\right)$be the space of all the Lebesgue measurable and 
$p$-integrable complex-valued functions defined on $\mathbb{R}^{+}$endowed with the usual norm $\|f\|_{p}$. Analogous notations we give for functions defined on $\mathbb{R}$.

For $p=1$, and $c \in \mathbb{R}$, let us consider the space

$$
X_{c}=\left\{f: \mathbb{R}^{+} \rightarrow \mathbb{C}: f(\cdot)(\cdot)^{c-1} \in L^{1}\left(\mathbb{R}^{+}\right)\right\}
$$

endowed with the norm

$$
\|f\|_{X_{c}}=\left\|f(\cdot)(\cdot)^{c-1}\right\|_{1}=\int_{0}^{\infty}|f(u)| u^{c-1} d u .
$$

More generally by $X_{c}^{p}$ we denote the space of all the functions $f: \mathbb{R}^{+} \rightarrow \mathbb{C}$ such that $f(\cdot)(\cdot)^{c-1 / p} \in L^{p}\left(\mathbb{R}^{+}\right)$, with $1<p<\infty$. In an equivalent form, $X_{c}^{p}$ is the space of all functions $f$ such that $(\cdot)^{c} f(\cdot) \in L_{\mu}^{p}\left(\mathbb{R}^{+}\right)$, where $L_{\mu}^{p}=L_{\mu}^{p}\left(\mathbb{R}^{+}\right)$ denotes the Lebesgue space with respect to the (invariant) measure

$$
\mu(A)=\int_{A} \frac{d t}{t}
$$

for any measurable set $A \subset \mathbb{R}^{+}$.

For $p=2$ we will denote by $\langle f, g\rangle_{2}$ the usual scalar product of the Hilbert space $L_{\mu}^{2}$ defined by

$$
\langle f, g\rangle_{2}:=\int_{0}^{\infty} f(u) \overline{g(u)} \frac{d u}{u} .
$$

Also, the space $X_{c}^{2}$ is a Hilbert space with respect to the scalar product:

$$
\langle f, g\rangle:=\int_{0}^{\infty} f(u) \overline{g(u)} u^{2 c} \frac{d u}{u} .
$$

The Mellin transform of a function $f \in X_{c}$ is defined by (see e.g. [21], [12])

$$
M[f](s) \equiv[f]_{M}^{\wedge}(s)=\int_{0}^{\infty} u^{s-1} f(u) d u, \quad(s=c+i t, t \in \mathbb{R}) .
$$

Basic properties of the Mellin transform are the following

$$
\begin{gathered}
M[a f(\cdot)+b g(\cdot)](s)=a M[f](s)+b M[g](s) \quad\left(f, g \in X_{c}, a, b \in \mathbb{R}\right) \\
|M[f(\cdot)](s)| \leq\|f\|_{X_{c}}(s=c+i t) .
\end{gathered}
$$

The inverse Mellin transform $M_{c}^{-1}[g]$ of the function $g \in L^{1}(\{c\} \times i \mathbb{R})$, is defined by:

$$
M_{c}^{-1}[g](x) \equiv M_{c}^{-1}[g(c+i t)](x):=\frac{x^{-c}}{2 \pi} \int_{-\infty}^{+\infty} g(c+i t) x^{-i t} d t, \quad\left(x \in \mathbb{R}^{+}\right),
$$

where by $L^{p}(\{c\} \times i \mathbb{R})$, for $p \geq 1$, we mean the space of all functions $g:\{c\} \times i \mathbb{R} \rightarrow$ $\mathbb{C}$ with $g(c+i \cdot) \in L^{p}\left(\mathbb{R}^{+}\right)$. 
More generally, for $1<p \leq 2$, we define the Mellin transform $M^{p}$ of $f \in X_{c}^{p}$, as (see [15], [14])

$$
M^{p}[f](s) \equiv[f]_{M^{p}}^{\wedge}(s)=\text { l.i.m. } \rho \rightarrow+\infty \int_{1 / \rho}^{\rho} f(u) u^{s-1} d u, \quad(s=c+i t),
$$

in the sense that

$$
\lim _{\rho \rightarrow \infty}\left\|M^{p}[f](c+i t)-\int_{1 / \rho}^{\rho} f(u) u^{s-1} d u\right\|_{L^{p}(\{c\} \times i \mathbb{R})}=0 .
$$

The Mellin translation operator $\tau_{h}^{c}$, for $h \in \mathbb{R}^{+}, c \in \mathbb{R}, f: \mathbb{R}^{+} \rightarrow \mathbb{C}$, is defined by

$$
\left(\tau_{h}^{c} f\right)(x):=h^{c} f(h x) \quad\left(x \in \mathbb{R}^{+}\right) .
$$

Setting $\tau_{h}:=\tau_{h}^{0}$, then $\left(\tau_{h}^{c} f\right)(x)=h^{c}\left(\tau_{h} f\right)(x),\left\|\tau_{h}^{c} f\right\|_{X_{c}}=\|f\|_{X_{c}}$.

The Mellin convolution product of two functions $f, g: \mathbb{R}^{+} \rightarrow \mathbb{C}$, denoted by $f * g$, is defined by

$$
(f * g)(x):=\int_{0}^{+\infty} g\left(\frac{x}{u}\right) f(u) \frac{d u}{u}=\int_{0}^{+\infty}\left(\tau_{1 / u}^{c} f\right)(x) g(u) u^{c} \frac{d u}{u}
$$

in case the integral exists. We have the following Lemma (for the proof see [12], Theorem 3)

Lemma 1. The Mellin convolution product has the following properties

(i) If $f, g \in X_{c}$, for $c \in \mathbb{R}$, then $f * g$ exists (a.e.) on $\mathbb{R}^{+}$, it belongs to $X_{c}$, and

$$
\|f * g\|_{X_{c}} \leq\|f\|_{X_{c}}\|g\|_{X_{c}} .
$$

If in addition $x^{c} f(x)$ is uniformly continuous on $\mathbb{R}^{+}$, then $f * g$ is continuous on $\mathbb{R}^{+}$.

(ii) (Convolution Theorem). If $f, g \in X_{c}$ and $s=c+i t, t \in \mathbb{R}$, then

$$
M[f * g](s)=M[f](s) M[g](s) .
$$

(iii) Commutativity and associativity of convolution product: for $f_{1}, f_{2}, f_{3} \in X_{c}$ there holds true (a.e.)

$$
f_{1} * f_{2}=f_{2} * f_{1}, \quad\left(f_{1} * f_{2}\right) * f_{3}=f_{1} *\left(f_{2} * f_{3}\right) .
$$

In particular $X_{c}$ is a Banach algebra.

We have the following preliminary results. The first one is proved in [12], Theorem 6 . 
Lemma 2 (Inversion Theorem). If $f \in X_{c}$ is such that $M[f] \in L^{1}(\{c\} \times i \mathbb{R})$, then

$$
M_{c}^{-1}[M[f]](x)=\frac{x^{-c}}{2 \pi} \int_{-\infty}^{\infty}[f]_{M}^{\wedge}(c+i t) x^{-i t} d t=f(x), \text { a.e. on } \mathbb{R}^{+} \text {. }
$$

Under the hypothesis that $f \in X_{c}$ and $M[f] \in L^{1}(\{c\} \times i \mathbb{R})$, the following lemma will enable us to work in Sec. 5 in a practical Hilbert space setting.

Lemma 3. If $f \in X_{c}$, with $c \in \mathbb{R}$, and $M[f] \in L^{1}(\{c\} \times i \mathbb{R})$, then $f \in X_{c}^{2}$.

Proof. By Lemma 2, we have

$$
f(x)=\frac{x^{-c}}{2 \pi} \int_{-\infty}^{+\infty}[f]_{M}^{\wedge}(c+i t) x^{-i t} d t, \text { a.e. } x \in \mathbb{R}^{+} .
$$

Then

$$
\left|x^{c} f(x)\right| \leq \frac{1}{2 \pi} \int_{-\infty}^{+\infty}\left|[f]_{M}^{\wedge}(c+i t)\right| d t \leq \frac{\left\|[f]_{M}^{\wedge}\right\|_{1}}{2 \pi}:=Q
$$

and so the function $x^{c} f(x)$ is essentially bounded. Thus

$$
\int_{0}^{\infty}\left|x^{c} f(x)\right|^{2} \frac{d x}{x} \leq Q \int_{0}^{\infty} x^{c}|f(x)| \frac{d x}{x}=Q\|f\|_{X_{c}} .
$$

So the assertion follows.

Another basic result is the following Mellin Parseval formula (see [12], Theorem 9):

Lemma 4. If $f, g \in X_{c}$, with $M[f] \in L^{1}(\{c\} \times i \mathbb{R})$ for some $c \in \mathbb{R}$, then

$$
\int_{0}^{\infty} f(u) g\left(\frac{x}{u}\right) \frac{d u}{u}=\frac{x^{-c}}{2 \pi} \int_{-\infty}^{+\infty}[f]_{M}^{\wedge}(c+i t)[g]_{M}^{\wedge}(c+i t) x^{-i t} d t .
$$

In what follows we will need a further tool in order to justify the interchange of integration and summation. In this respect the following Lemma concerning the spaces $L_{\mu}^{2}\left(\mathbb{R}^{+}\right)$will be useful (for usual Lebesgue spaces $L^{p}(\mathbb{R})$, see Lemma 2.3 in $[9])$.

Lemma 5. Let $f, g \in L_{\mu}^{2}\left(\mathbb{R}^{+}\right)$, and let $\left(f_{n}\right)_{n \in \mathbb{N}},\left(g_{n}\right)_{n \in \mathbb{N}} \subset L_{\mu}^{2}\left(\mathbb{R}^{+}\right)$be two sequences which are convergent in $L_{\mu}^{2}-$ norm to $f, g$ respectively. Then we have

$$
\lim _{n \rightarrow+\infty} \int_{0}^{\infty} f_{n}(u) \overline{g_{n}(u)} \frac{d u}{u}=\int_{0}^{\infty} f(u) \overline{g(u)} \frac{d u}{u} .
$$

The proof follows the same lines of Lemma 2.3 in [9]. 


\section{The Strong Mellin Fractional Differential Operator}

The Mellin fractional difference of $f \in X_{c}$ of order $\alpha>0$, with increment $h>0$ is defined by

$$
\Delta_{h}^{\alpha, c} f(x):=\left(\tau_{h}^{c}-I\right)^{\alpha} f(x)=\sum_{j=0}^{\infty}\left(\begin{array}{c}
\alpha \\
j
\end{array}\right)(-1)^{\alpha-j} \tau_{h^{j}}^{c} f(x),
$$

$I$ being the identity operator over the space of all measurable functions on $\mathbb{R}^{+}$, and

$$
\left(\begin{array}{c}
\alpha \\
j
\end{array}\right)=\frac{\alpha(\alpha-1) \cdots(\alpha-j+1)}{j !} .
$$

It has the following properties

Proposition 1. For $f \in X_{c}$ the difference $\Delta_{h}^{\alpha, c} f(x)$ exists a.e. for $h>0$, with
i) $\left\|\Delta_{h}^{\alpha, c} f\right\|_{X_{c}} \leq\|f\|_{X_{c}} \sum_{j=0}^{\infty}\left|\left(\begin{array}{c}\alpha \\ j\end{array}\right)\right|$,
ii) $M\left[\Delta_{h}^{\alpha, c} f\right](c+i t)=\left(h^{-i t}-1\right)^{\alpha} M[f](c+i t)$.

Proof. (i) follows immediately by the definitions of the differences, while (ii) follows by taking the Mellin transforms on the left of (3), thus

$$
\sum_{j=0}^{\infty}\left(\begin{array}{c}
\alpha \\
j
\end{array}\right)(-1)^{\alpha-j} h^{-i t j} M[f](c+i t) .
$$

If for $f \in X_{c}$ there exists $g \in X_{c}$ such that

$$
\lim _{h \rightarrow 1}\left\|\frac{\Delta_{h}^{\alpha, c} f(x)}{(h-1)^{\alpha}}-g(x)\right\|_{X_{c}}=0
$$

then $g$ is called the strong fractional derivative of $f$ of order $\alpha$, it is denoted by $g(x)=\mathrm{s}-\Theta_{c}^{\alpha} f(x)$, and

$$
W_{X_{c}}^{\alpha}:=\left\{f \in X_{c}: \mathrm{s}-\Theta_{c}^{\alpha} f \text { exists and s- } \Theta_{c}^{\alpha} f \in X_{c}\right\},
$$

is the Mellin Sobolev space. For $\alpha=0$ we put $W_{X_{c}}^{0}=X_{c}$.

Theorem 1. If $f \in W_{X_{c}}^{\alpha}$, then for $s=c+i t, t \in \mathbb{R}$,

$$
M\left[s-\Theta_{c}^{\alpha} f\right](s)=(-i t)^{\alpha} M[f](s) .
$$


Proof. The assertion follows in view of

$$
\lim _{h \rightarrow 1}\left(\frac{h^{-i t}-1}{h-1}\right)^{\alpha}=(-i t)^{\alpha}
$$

so that we can deduce

$$
\lim _{h \rightarrow 1}\left|(-i t)^{\alpha}[f]_{M}^{\wedge}(s)-\left[\mathrm{s}-\Theta_{c}^{\alpha} f\right]_{M}^{\wedge}(s)\right|=0
$$

The above definition of strong derivatives is inspired by the analogous definition for integral values of $\alpha$, introduced and studied in [11], along with its equivalence with the concept of a pointwise Mellin derivatives, also studied there, for functions belonging to the space $W_{X_{c}}^{\alpha}$, (see Theorem 4.3 in [11]). For the material of this section and for further related results see [5]. In particular, there, a concept of a pointwise fractional derivative, first introduced in [15], is discussed, along with the full equivalence between the two concepts (see Section 4.2 and Theorem 5 in [5]).

\section{The Sampling Theorem and the Reproducing Ker- nel Formula in the Mellin Setting}

\subsection{Exponential Sampling Formula}

Let $B_{c, T}^{1}$ denote the class of functions $f \in X_{c}, f \in C\left(\mathbb{R}^{+}\right), c \in \mathbb{R}$, which are Mellin band-limited in the interval $[-T, T], T \in \mathbb{R}^{+}$, thus for which $[f]_{M}^{\wedge}(c+i t)=$ 0 for all $|t|>T$. By Lemma 3 we have $B_{c, T}^{1} \subset X_{c}^{2}$. A mathematician's version of the exponential sampling theorem, introduced by the electrical engineers/physicists M.Bertero, E.R. Pike [8] and F. Gori [18] (see also [20]) and proven in [13], Theorem 6.3, reads as follows

Theorem 2 (Exponential Sampling Formula (ESF)). If $f \in B_{c, \pi T}^{1}$ for some $c \in \mathbb{R}$, and $T>0$, then the series

$$
x^{c} \sum_{k=-\infty}^{\infty} f\left(e^{k / T}\right) \operatorname{lin}_{c / T}\left(e^{-k} x^{T}\right)
$$

is uniformly convergent in $\mathbb{R}^{+}$, and one has the representation

$$
f(x)=\sum_{k=-\infty}^{\infty} f\left(e^{k / T}\right) \operatorname{lin}_{c / T}\left(e^{-k} x^{T}\right) \equiv E_{T}^{c} f(x) \quad\left(x \in \mathbb{R}^{+}\right) .
$$


The $\operatorname{lin}_{c}$-function for $c \in \mathbb{R}, \operatorname{lin}_{c}: \mathbb{R}^{+} \rightarrow \mathbb{R}$, is defined, for $x \in \mathbb{R}^{+} \backslash\{1\}$, by

$$
\operatorname{lin}_{c}(x)=\frac{x^{-c}}{2 \pi i} \frac{x^{\pi i}-x^{-\pi i}}{\log x}=\frac{x^{-c}}{2 \pi} \int_{-\pi}^{\pi} x^{-i t} d t
$$

with the continuous extension $\operatorname{lin}_{c}(1):=1$, thus $\operatorname{lin}_{c}(x)=x^{-c} \operatorname{sinc}(\log x)$.

It is clear that $\operatorname{lin}_{c} \notin X_{\bar{c}}$ for any $\bar{c}$. However, it belongs to the space $X_{c}^{2}$ and its Mellin transform in $X_{c}^{2}$-sense is given by

$$
M^{2}\left[\operatorname{lin}_{c}\right](c+i t)=\chi_{[-\pi, \pi]}(t), \quad t \in \mathbb{R} .
$$

The above exponential sampling theorem can also be established for bandlimited functions in $X_{c}^{2}$. We denote by $B_{c, \pi T}^{2}$ the space of all functions in $X_{c}^{2}$ such that $[f]_{M^{2}}^{\wedge}(c+i t)=0$ for all $|t|>\pi T$. It is known that $B_{c, \pi T}^{1} \subset B_{c, \pi T}^{2}$. Using the Mellin transform of $f \in X_{c}^{2}$, the same statement as that of Theorem 2 is shown in [14].

In the following, we will use the following two properties of the $\operatorname{lin}_{c}-$ functions.

Lemma 6. We have, for every $x, y>0, c \in \mathbb{R}, T>0$

$$
\operatorname{lin}_{c / T}\left(\left(\frac{x}{y}\right)^{T}\right)=\left(\frac{y}{x}\right)^{2 c} \operatorname{lin}_{c / T}\left(\left(\frac{y}{x}\right)^{T}\right)
$$

Proof. Indeed this easily follows from (5), since the integral representations of both sides of the equality are exactly the same.

Lemma 7. We have the following "lin" summation formula, for every $x, y \in \mathbb{R}^{+}$

$$
\sum_{k=-\infty}^{+\infty} \operatorname{lin}_{c / T}\left(e^{k} y^{-T}\right) \operatorname{lin}_{c / T}\left(e^{-k} x^{T}\right)=\left(\frac{y}{x}\right)^{2 c} \operatorname{lin}_{c / T}\left(\left(\frac{y}{x}\right)^{T}\right)
$$

Proof. Using the sinc summation formula (see [9], Formula (20)), namely

$$
\operatorname{sinc}(\alpha-\beta)=\sum_{k=-\infty}^{\infty} \operatorname{sinc}(\alpha-k) \operatorname{sinc}(\beta-k) \quad(\alpha, \beta \in \mathbb{C})
$$


we have

$$
\begin{aligned}
& \sum_{k=-\infty}^{+\infty} \operatorname{lin}_{c / T}\left(e^{k} y^{-T}\right) \operatorname{lin}_{c / T}\left(e^{-k} x^{T}\right) \\
= & \sum_{k=-\infty}^{+\infty}\left(e^{-k} y^{-T}\right)^{-c / T} \operatorname{sinc}(T \log x-k)\left(e^{-k} x^{T}\right)^{-c / T} \operatorname{sinc}(T \log x-k) \\
= & \left(\frac{y}{x}\right)^{c} \sum_{k=-\infty}^{+\infty} \operatorname{sinc}(T \log y-k) \operatorname{sinc}(T \log x-k)=\left(\frac{y}{x}\right)^{c} \operatorname{sinc}\left(\log \left(\left(\frac{y}{x}\right)^{T}\right)\right. \\
= & \left(\frac{y}{x}\right)^{c}\left(\left(\frac{y}{x}\right)^{T}\right)^{c / T}\left(\left(\frac{y}{x}\right)^{T}\right)^{-c / T} \operatorname{sinc}\left(\log \left(\left(\frac{y}{x}\right)^{T}\right)=\left(\frac{y}{x}\right)^{2 c} \operatorname{lin}_{c / T}\left(\left(\frac{y}{x}\right)^{T}\right)\right.
\end{aligned}
$$

As we all know, bandlimitation in the classical Fourier version of the WhittakerKotel'nikov-Shannon sampling theorem is a restriction we try to avoid. Likewise it is so in the Mellin setting. In this respect we have the following approximate version (see [13], Theorem 7.2).

Theorem 3 (Approximate Exponential Sampling Formula (AESF)). Let $f \in$ $X_{c} \cap C\left(\mathbb{R}^{+}\right), c \in \mathbb{R}$, be such that $M[f] \in L^{1}(\{c\} \times i \mathbb{R})$. Then there holds the error estimate

$$
\begin{aligned}
& \left|f(x)-\sum_{k=-\infty}^{\infty} f\left(e^{k / T}\right) \operatorname{lin}_{c / T}\left(e^{-k} x^{T}\right)\right| \\
\leq & \frac{x^{-c}}{\pi} \int_{|t|>\pi T}|M[f](c+i t)| d t \quad\left(x \in \mathbb{R}^{+}, T>0\right) .
\end{aligned}
$$

Corollary 1. Let $f \in X_{c} \cap C\left(\mathbb{R}^{+}\right), c \in \mathbb{R}$, be such that $M[f] \in L^{1}(\{c\} \times i \mathbb{R})$. Then

$$
\lim _{T \rightarrow+\infty} x^{c}\left|f(x)-E_{T}^{c} f(x)\right|=0, \quad\left(x \in \mathbb{R}^{+}\right) .
$$

Further, if $f \in B_{c, \pi \bar{T}}^{1}$ for some $\bar{T}>0$, then, for all $T \geq \bar{T}$,

$$
f(x)=E_{T}^{c} f(x), \quad\left(x \in \mathbb{R}^{+}\right) .
$$

The operator s- $\Theta_{c}^{\alpha} f, \alpha>0$, plays the basic role in the following new corollary, giving the fast rate of approximation of $f(x)$, depending on its order $\alpha$, by the exponential sampling sum $E_{T}^{c} f(x)$. 
Corollary 2. Let $f \in X_{c} \cap C\left(\mathbb{R}^{+}\right)$, be such that $M[f] \in L^{1}(\{c\} \times i \mathbb{R})$ for some $c \in \mathbb{R}$. Then

$f(x)=\lim _{T \rightarrow+\infty} \frac{x^{-c}}{2 \pi} \int_{-T}^{T}[f]_{M}^{\wedge}(c+i t) x^{-i t} d t=\frac{x^{-c}}{2 \pi} \int_{-\infty}^{\infty}[f]_{M}^{\wedge}(c+i t) x^{-i t} d t \quad\left(x \in \mathbb{R}^{+}\right)$.

If in addition $f \in W_{X_{c}}^{\alpha}, c \in \mathbb{R}, \alpha>0$, is such that $M\left[s-\Theta_{c}^{\alpha} f\right] \in L^{1}(\{c\} \times i \mathbb{R})$, then $f$ is approximately Mellin band-limited in the sense that

$$
\left|f(x)-\frac{x^{-c}}{2 \pi} \int_{-T \pi}^{T \pi}[f]_{M}^{\wedge}(c+i t) x^{-i t} d t\right|=o\left(T^{-\alpha}\right) \quad\left(T \rightarrow+\infty, x \in \mathbb{R}^{+}\right),
$$

and $f$ has the same rate of approximation by $E_{T}^{c} f$, namely

$$
x^{c}\left|f(x)-E_{T}^{c} f(x)\right|=o\left(T^{-\alpha}\right) \quad\left(T \rightarrow+\infty ; x \in \mathbb{R}^{+}\right) .
$$

Proof. The first part of the proof follows by Lemma 2. The second part follows from the estimate of Theorem 3, noting

$$
\left|[f]_{M}^{\wedge}(c+i t)\right|=|t|^{-\alpha}\left|\left[\mathrm{s}-\Theta_{c}^{\alpha} f\right]_{M}^{\wedge}(c+i t)\right|, t \in \mathbb{R},
$$

by Theorem 1, so that:

$$
x^{-c} \int_{|t|>\pi T}\left|[f]_{M}^{\wedge}(c+i t)\right| d t \leq \frac{x^{-c}}{\pi^{\alpha} T^{\alpha}} \int_{|t|>\pi T}\left|\left[\mathrm{~s}-\Theta_{c}^{\alpha} f\right]_{M}^{\wedge}(c+i t)\right| d t=o\left(T^{-\alpha}\right) .
$$

\subsection{Mellin reproducing kernel formula}

One of the several theorems which are equivalent to the classical WhittakerKotel'nikov-Shannon sampling theorem is the well known reproducing kernel formula (see [9]). In [4] we announced a Mellin version setting of this result for functions in $B_{c}^{\pi T}, T>0$, also giving a short sketch of the proof. Here we give a detailed proof of it.

Theorem 4 (Mellin Reproducing Kernel Formula (MRKF)). For $f \in B_{c, \pi T}^{1}$, with $c \in \mathbb{R}$ and $T>0$, we have

$$
f(x)=T \int_{0}^{\infty} f(y) \operatorname{lin}_{c / T}\left(\left(\frac{x}{y}\right)^{T}\right) \frac{d y}{y},
$$

the integral being absolutely convergent in $\mathbb{R}^{+}$. 
Proof. The above integral is absolutely convergent, since $f \in X_{c}$ and we have

$$
\left|\operatorname{lin}_{c / T}(x)\right| \leq x^{-c / T}|\operatorname{sinc}(\log x)| \leq x^{-c / T},
$$

for every $x \in \mathbb{R}^{+}$. Putting $h(y)=f\left(y^{1 / T}\right)$, we have $h \in B_{c / T, \pi}^{1}$. Moreover, the function $h(y) \operatorname{lin}_{c / T}(x / y)$ is continuous and it belongs to $X_{0}$ as a function of $y$. Indeed we have

$$
\left\|h(\cdot) \operatorname{lin}_{c / T}(x /(\cdot))\right\|_{X_{0}} \leq \frac{T}{x^{c / T}}\|f\|_{X_{c}} .
$$

Now, using the reasoning of Lemma 6.3 in [13], page 115, with $c / T$ in place of $c$, we can write

$$
\left[h(y) \operatorname{lin}_{c / T}(x / y)\right]_{M}^{\wedge}(i t)=\frac{x^{-c / T}}{2 \pi} \int_{-\pi}^{\pi}[h]_{M}^{\wedge}(c / T+i(t+v)) x^{-i v} d v \quad(t \in \mathbb{R}) .
$$

Then for $t=0$ we obtain

$$
\int_{0}^{\infty} h(y) \operatorname{lin}_{c / T}(x / y) \frac{d y}{y}=\frac{x^{-c / T}}{2 \pi} \int_{-\pi}^{\pi}[h]_{M}^{\wedge}(c / T+i v) x^{-i v} d v=h(x)
$$

by Lemma 2. Thus we have

$$
f\left(x^{1 / T}\right)=T \int_{0}^{\infty} f(y) \operatorname{lin}_{c / T}\left(x / y^{T}\right) \frac{d y}{y},
$$

and putting $x^{1 / T}=z$ we have the assertion.

Next, using the Mellin transform of $X_{c}^{2}$ functions, we prove the MRKF formula also for functions in $B_{c, \pi T}^{2}$.

Theorem 5 (Mellin Reproducing Kernel Formula in $X_{c}^{2}$ ). For $f \in B_{c, \pi T}^{2}$, with $c \in \mathbb{R}$ and $T>0$, we have

$$
f(x)=T \int_{0}^{\infty} f(y) \operatorname{lin}_{c / T}\left(\left(\frac{x}{y}\right)^{T}\right) \frac{d y}{y},
$$

the integral being absolutely convergent in $\mathbb{R}^{+}$.

Proof. The above integral is absolutely convergent in view of the definition of the $\operatorname{lin}_{c}$-function, the fact that $f \in X_{c}^{2}$ and the Hölder inequality in $L_{\mu}^{2}$. Indeed,

$$
\begin{aligned}
& \int_{0}^{\infty}|f(y)|\left|\operatorname{lin}_{c / T}\left(\left(\frac{x}{y}\right)^{T}\right)\right| \frac{d y}{y}=\int_{0}^{\infty}|f(y)|\left(\frac{x}{y}\right)^{-c}\left|\operatorname{sinc}\left(T \log \left(\frac{x}{y}\right)\right)\right| \frac{d y}{y} \\
\leq & x^{-c}\left(\int_{0}^{\infty}|f(y)|^{2} y^{2 c} \frac{d y}{y}\right)^{1 / 2}\left(\int_{0}^{\infty}\left|\operatorname{sinc}\left(T \log \left(\frac{x}{y}\right)\right)\right|^{2} \frac{d y}{y}\right)^{1 / 2} \\
\leq & \frac{x^{-c}}{T}\|f\|_{X_{c}^{2}}\|\operatorname{sinc}\|_{L^{2}(\mathbb{R})} .
\end{aligned}
$$


Putting, as before, $h(y)=f\left(y^{1 / T}\right)$, we have $h \in B_{c / T, \pi}^{2}$. Moreover, the function $h(y) \operatorname{lin}_{c / T}(x / y)$ is continuous and belongs to $X_{0}^{2}$ as a function of $y$. Indeed we have

$$
\left\|h(\cdot) \operatorname{lin}_{c / T}(x /(\cdot))\right\|_{X_{0}^{2}} \leq \frac{T^{1 / 2}}{x^{c / T}}\|f\|_{X_{c}^{2}} .
$$

Now, putting for any function $g$ and $\rho>1$,

$$
\left[g_{\rho}\right]_{M^{2}}^{\wedge}(i t):=\int_{1 / \rho}^{\rho} g(y) y^{i t} \frac{d y}{y},
$$

we have that

$$
\left[\left(h(y) \operatorname{lin}_{c / T}(x / y)\right)_{\rho}\right]_{M^{2}}^{\wedge}(i t)=\frac{x^{-c / T}}{2 \pi} \int_{-\pi}^{\pi}\left[h_{\rho}\right]_{M^{2}}^{\wedge}(c / T+i(t+v)) x^{-i v} d v \quad(t \in \mathbb{R}) .
$$

Thus, taking also into account that $[h]_{M^{2}}^{\wedge} \in L^{1}(i \mathbb{R})$, by a Minkowski type inequality we have

$$
\begin{aligned}
& \left\|\frac{x^{-c / T}}{2 \pi} \int_{-\pi}^{\pi}[h]_{M^{2}}^{\wedge}(c / T+i((\cdot)+v)) x^{-i v} d v-\left[\left(h(y) \operatorname{lin}_{c / T}(x / y)\right)_{\rho}\right]_{M^{2}}^{\wedge}(i(\cdot))\right\|_{L^{2}(i \mathbb{R})} \\
\leq & \frac{x^{-c / T}}{2 \pi} \int_{-\pi}^{\pi} \|[h]_{M^{2}}^{\wedge}\left(c / T+i((\cdot)+v)-\left[h_{\rho}\right]_{M^{2}}^{\wedge}\left(c / T+i((\cdot)+v) \|_{L^{2}(i \mathbb{R})} d v\right.\right.
\end{aligned}
$$

Passing, if necessary, to subsequences $\left(\rho_{n}\right)$ with $\rho_{n} \rightarrow+\infty$, we get,

$$
\lim _{n \rightarrow+\infty}\left[\left(h(y) \operatorname{lin}_{c / T}(x / y)\right)_{\rho_{n}}\right]_{M^{2}}^{\wedge}(i t)=\frac{x^{-c / T}}{2 \pi} \int_{-\pi}^{\pi}[h]_{M^{2}}^{\wedge}(c / T+i(t+v)) x^{-i v} d v \text {, a.e. } t \in \mathbb{R} \text {. }
$$

By the asbolute convergence of the integral in (6), we can write the above limit relation as

$$
\int_{0}^{\infty} h(y) \operatorname{lin}_{c / T}(x / y) y^{i t} \frac{d y}{y}=\frac{x^{-c / T}}{2 \pi} \int_{-\pi}^{\pi}[h]_{M^{2}}^{\wedge}(c / T+i(t+v)) x^{-i v} d v, \quad \text { a.e. } t \in \mathbb{R} .
$$

Since the right-hand side of the previous relation is a continuous function of $t$, the result hold for any $t \in \mathbb{R}$, and taking $t=0$ we obtain, by Lemma 5.1 in [14] (namely Lemma 2 for $X_{c}^{2}$, in the band-limited case)

$$
\int_{0}^{\infty} h(y) \operatorname{lin}_{c / T}(x / y) \frac{d y}{y}=\frac{x^{-c / T}}{2 \pi} \int_{-\pi}^{\pi}[h]_{M}^{\wedge}(c / T+i v) x^{-i v} d v=h(x) .
$$

Thus we have

$$
f\left(x^{1 / T}\right)=T \int_{0}^{\infty} f(y) \operatorname{lin}_{c / T}\left(x / y^{T}\right) \frac{d y}{y},
$$

and putting $x^{1 / T}=z$ we have the assertion.

As an important consequence of Theorem 5, is the following orthogonality property of the $\operatorname{lin}_{c}$-functions: 
Corollary 3. We have, for $j, k \in \mathbb{Z}$,

$$
T e^{-2 c k / T}\left\langle\operatorname{lin}_{c / T}\left((\cdot)^{T} e^{-j}\right), \operatorname{lin}_{c / T}\left((\cdot)^{T} e^{-k}\right)\right\rangle=\operatorname{lin}_{c / T}\left(e^{k-j}\right)=\delta_{j, k},
$$

where $\delta_{j, k}$ is the Kronecker symbol.

Proof. By Theorem 5 and Lemma 6 we can write,

$$
\begin{aligned}
f(x) & =T \int_{0}^{\infty} f(y) \operatorname{lin}_{c / T}\left(\left(\frac{x}{y}\right)^{T}\right) \frac{d y}{y}=T \int_{0}^{\infty} f(y)\left(\frac{y}{x}\right)^{2 c} \operatorname{lin}_{c / T}\left(\left(\frac{y}{x}\right)^{T}\right) \frac{d y}{y} \\
& =T x^{-2 c}\left\langle f, \operatorname{lin}_{c / T}\left(\left(\frac{(\cdot)}{x}\right)^{T}\right)\right\rangle
\end{aligned}
$$

and so for $x=e^{k / T}, k \in \mathbb{Z}$, we get

$$
f\left(e^{k / T}\right)=T e^{-2 c k / T}\left\langle f, \operatorname{lin}_{c / T}\left((\cdot)^{T} e^{-k}\right)\right\rangle .
$$

The assertion follows taking $f(x)=\operatorname{lin}_{c / T}\left(x^{T} e^{-j}\right)$, for $j \in \mathbb{Z}$, noting that its Mellin transform is supported on the interval $[-\pi T, \pi T]$.

As in Fourier analysis there holds the approximate version of the above reproducing kernel formula, see [10]. For not necessarily Mellin bandlimited functions it reads

Theorem 6 (Approximate Mellin Reproducing Kernel Formula (AMRKF)). If $f \in X_{c} \cap C\left(\mathbb{R}^{+}\right)$with $c \in \mathbb{R}$, and $M[f] \in L^{1}(\{c\} \times i \mathbb{R})$, then there holds, for $x \in \mathbb{R}^{+}$, and $T>0$,

$$
f(x)=T \int_{0}^{\infty} f(y) \operatorname{lin}_{c / T}\left(\left(\frac{x}{y}\right)^{T}\right) \frac{d y}{y}+\left(R_{\pi T} f\right)(x)
$$

where the aliasing error

$$
\left(R_{\pi T} f\right)(x)=\frac{x^{-c}}{2 \pi} \int_{|t| \geq \pi T}[f]_{M}^{\wedge}(c+i t) x^{-i t} d t .
$$

Furthermore we have the error estimate

$$
\left|\left(R_{\pi T} f\right)(x)\right| \leq \frac{x^{-c}}{2 \pi} \int_{|t| \geq \pi T}\left|[f]_{M}^{\wedge}(c+i t)\right| d t
$$

Proof. Firstly, by (5), putting $G(x):=\operatorname{lin}_{c / T}\left(x^{T}\right)$, we have

$$
G(x)=\frac{x^{-c}}{2 \pi} \int_{-\pi}^{\pi} x^{-i t T} d t=\frac{x^{-c}}{2 \pi} \int_{-\pi T}^{\pi T} x^{-i u} d u
$$


and so the $L^{2}-$ Mellin transform of $G$ is given by

$$
[G]_{M^{2}}^{\wedge}(v)=\frac{1}{T} \chi_{[-\pi T, \pi T]}(u)
$$

Therefore, by Lemma 4 we get

$$
\begin{aligned}
& T \int_{0}^{\infty} f(y) \operatorname{lin}_{c / T}\left(\left(\frac{x}{y}\right)^{T}\right) \frac{d y}{y} \\
= & \frac{x^{-c}}{2 \pi} \int_{-\infty}^{+\infty}[f]_{M}^{\wedge}(c+i t)[G]_{M}^{\wedge}(c+i t) x^{-i t} d t=\frac{x^{-c}}{2 \pi} \int_{-\pi T}^{\pi T}[f]_{M}^{\wedge}(c+i t) x^{-i t} d t .
\end{aligned}
$$

By the inversion formula (3), the latter integral equals $f(x)$ for every $x \in \mathbb{R}$. So, finally

$$
f(x)-T \int_{0}^{\infty} f(y) \operatorname{lin}_{c / T}\left(\left(\frac{x}{y}\right)^{T}\right) \frac{d y}{y}=\frac{x^{-c}}{2 \pi} \int_{|t| \geq \pi T}[f]_{M}^{\wedge}(c+i t) x^{-i t} d t .
$$

Thus the assertion follows and the error estimate is obvious.

Corollary 4. If $f \in X_{c} \cap C\left(\mathbb{R}^{+}\right), c \in \mathbb{R}$, such that $M[f] \in L^{1}(\{c\} \times i \mathbb{R})$, then

$$
\lim _{T \rightarrow+\infty} x^{-c}\left|\left(R_{\pi T} f\right)(x)\right|=0 \quad\left(x \in \mathbb{R}^{+}\right) .
$$

Further, if $f \in B_{c, \pi \bar{T}}^{1}$, some $\bar{T}>0$, then for all $T>\bar{T}$

$$
\left|\left(R_{\pi T} f\right)(x)\right|=0 \quad\left(x \in \mathbb{R}^{+}\right) .
$$

Corollary 5. If $f \in W_{X_{c}}^{\alpha} \cap C\left(\mathbb{R}^{+}\right), c \in \mathbb{R}, \alpha>0$ such that $M\left[s-\Theta_{c}^{\alpha} f\right] \in$ $L^{1}(\{c\} \times i \mathbb{R})$, then

$$
x^{-c}\left|\left(R_{\pi T} f\right)(x)\right|=o\left(T^{-\alpha}\right) \quad\left(x \in \mathbb{R}^{+}, T \rightarrow+\infty\right) .
$$

The proofs are clear.

It is our conjecture that MRKF and its approximate version AMRKF are equivalent. The conjecture sounds incredible but is based on the fact that the classical WKS-sampling theorem is equivalent to its classical approximate version, as was very recently shown in [10]. Likewise the ESF could be equivalent to the AESF. If both conjectures turn out to be valid, then their proofs will probably be very tricky. Both results would be of essential interest in applications. 


\section{Interconnections between ESF and MRKF for Mellin- bandlimited Signals}

In this section we prove the interconnections between the ESF and MRKF, announced in [4]. We begin with:

Theorem 7. Let us consider $f \in B_{c, \pi T}^{1}$, for some $c \in \mathbb{R}, T>0$. Then $(E S F) \Rightarrow$ $(M R K F)$

Proof. Let $x, u \in \mathbb{R}^{+}$. We write the exponential formula (ESF) for the function $g(x)=f\left(x u^{1 / T}\right)$, which is again in $B_{c, \pi T}^{1}$, namely

$$
g(x)=\sum_{k=-\infty}^{+\infty} g\left(e^{k / T}\right) \operatorname{lin}_{c / T}\left(e^{-k} x^{T}\right)
$$

and use the properties of the Mellin transform, to deduce

$$
f\left(x u^{1 / T}\right)=\sum_{k=-\infty}^{+\infty} f\left(e^{k / T} u^{1 / T}\right) \operatorname{lin}_{c / T}\left(e^{-k} x^{T}\right) .
$$

Subsituting $x$ by $x u^{-1 / T}$, we obtain

$$
f(x)=\sum_{k=-\infty}^{+\infty} f\left(e^{k / T} u^{1 / T}\right) \operatorname{lin}_{c / T}\left(e^{-k} x^{T} u^{-1}\right) .
$$

Integrating in the Mellin sense both sides of this equality in the interval $[1, e]$, with respect to $u$, we get

$$
f(x)=\int_{1}^{e} \sum_{k=-\infty}^{+\infty} f\left(e^{k / T} u^{1 / T}\right) \operatorname{lin}_{c / T}\left(e^{-k} x^{T} u^{-1}\right) \frac{d u}{u} .
$$

Since the above series converges uniformly with respect to $u$ in the interval $[1, e]$, (the multiplication with the weight $x^{c}$ being now not essential), integration and summation may be interchanged, so obtaining

$$
f(x)=\sum_{k=-\infty}^{+\infty} \int_{1}^{e} f\left(e^{k / T} u^{1 / T}\right) \operatorname{lin}_{c / T}\left(e^{-k} x^{T} u^{-1}\right) \frac{d u}{u} .
$$

Putting $u^{1 / T} e^{k / T}=y$, we obtain the MRKF, thus

$f(x)=T \sum_{k=-\infty}^{+\infty} \int_{e^{k / T}}^{e^{(k+1) / T}} f(y) \operatorname{lin}_{c / T}\left(\left(\frac{x}{y}\right)^{T}\right) \frac{d y}{y}=T \int_{0}^{+\infty} f(y) \operatorname{lin}_{c / T}\left(\left(\frac{x}{y}\right)^{T}\right) \frac{d y}{y}$. 
Theorem 8. Let $f \in X_{c}^{2}$. Then $(M R K F) \Rightarrow(E S F)$.

Proof. Proceeding formally, we have now, by Lemmas 6 and 7 and by an interchange of integration and summation,

$$
\begin{aligned}
& f(x)=T \int_{0}^{\infty} f(y) \operatorname{lin}_{c / T}\left(\left(\frac{x}{y}\right)^{T}\right) \frac{d y}{y}=T \int_{0}^{\infty} f(y)\left(\frac{y}{x}\right)^{2 c} \operatorname{lin}_{c / T}\left(\left(\frac{y}{x}\right)^{T}\right) \frac{d y}{y} \\
= & T \int_{0}^{+\infty} f(y)\left[\sum_{k=-\infty}^{+\infty} \operatorname{lin}_{c / T}\left(e^{k} y^{-T}\right) \operatorname{lin}_{c / T}\left(e^{-k} x^{T}\right)\right] \frac{d y}{y} \\
= & T \sum_{k=-\infty}^{+\infty}\left[\int_{0}^{+\infty} f(y) \operatorname{lin}_{c / T}\left(e^{k} y^{-T}\right) \frac{d y}{y}\right] \operatorname{lin}_{c / T}\left(e^{-k} x^{T}\right)=\sum_{k=-\infty}^{+\infty} f\left(e^{k / T}\right) \operatorname{lin}_{c / T}\left(e^{-k} x^{T}\right)
\end{aligned}
$$

thus two applications of the MRKF would lead to the ESF. Now to a precise proof. First, note that

$$
G_{x}(\cdot):=\operatorname{lin}_{c / T}\left(\left(\frac{(\cdot)}{x}\right)^{T}\right) \in X_{c}^{2}
$$

Indeed, we have

$$
\begin{aligned}
& \int_{0}^{\infty} y^{2 c} \operatorname{lin}_{c / T}^{2}\left(\left(\frac{y}{x}\right)^{T}\right) \frac{d y}{y}=\int_{0}^{\infty} y^{2 c}\left(\left(\frac{y}{x}\right)^{T}\right)^{-2 c / T} \operatorname{sinc}^{2}\left(T \log \frac{y}{x}\right) \frac{d y}{y} \\
= & x^{2 c} \int_{0}^{\infty} \operatorname{sinc}^{2}\left(T \log \frac{y}{x}\right) \frac{d y}{y} .
\end{aligned}
$$

Now, putting $\log (y / x)=t$, we get

$$
\int_{0}^{\infty} y^{2 c} \operatorname{lin}_{c / T}^{2}\left(\left(\frac{y}{x}\right)^{T}\right) \frac{d y}{y}=x^{2 c} \int_{-\infty}^{\infty} \operatorname{sinc}^{2}(T t) d t<+\infty
$$

We have to justify the interchange in the above formal proof. Firstly, we prove the convergence of the exponential sampling series. Now, using the orthogonality property of $\operatorname{lin}_{c}$ functions (7), we can write

$$
\begin{aligned}
& \| f-\sum_{|k| \leq n} f\left(e^{k / T} \operatorname{lin}_{c / T}\left(e^{-k}(\cdot)^{T}\right) \|_{X_{c}^{2}}^{2}\right. \\
= & \left\langle f-\sum_{|j| \leq n} f\left(e^{j / T} \operatorname{lin}_{c / T}\left(e^{-j}(\cdot)^{T}\right), f-\sum_{|k| \leq n} f\left(e^{k / T} \operatorname{lin}_{c / T}\left(e^{-k}(\cdot)^{T}\right)\right\rangle\right.\right. \\
= & \|f\|_{X_{c}^{2}}^{2}-\sum_{|k| \leq n} \overline{f\left(e^{k / T}\right)}\left\langle f, \operatorname{lin}_{c / T}\left(e^{-k}(\cdot)^{T}\right)\right\rangle-\sum_{|j| \leq n} f\left(e^{j / T}\right) \overline{\left\langle f, \operatorname{lin}_{c / T}\left(e^{-j}(\cdot)^{T}\right)\right\rangle}+
\end{aligned}
$$




$$
\begin{aligned}
& +\sum_{|j| \leq n} \sum_{|k| \leq n} \overline{f\left(e^{k / T}\right)} f\left(e^{j / T}\right)\left\langle\operatorname{lin}_{c / T}\left(e^{-j}(\cdot)^{T}\right), \operatorname{lin}_{c / T}\left(e^{-k}(\cdot)^{T}\right)\right\rangle \\
& =\|f\|_{X_{c}^{2}}^{2}-\sum_{|k| \leq n} \overline{f\left(e^{k / T}\right)} \frac{1}{T} e^{2 c k / T} f\left(e^{k / T}\right)-\sum_{|j| \leq n} \overline{f\left(e^{j / T}\right)} \frac{1}{T} e^{2 c j / T} f\left(e^{j / T}\right) \\
& +\sum_{|k| \leq n} \overline{f\left(e^{k / T}\right)} \frac{1}{T} e^{2 c k / T} f\left(e^{k / T}\right)=\|f\|_{X_{c}^{2}}^{2}-\sum_{|j| \leq n}\left|f\left(e^{j / T}\right)\right|^{2} \frac{1}{T} e^{2 c j / T} \geq 0,
\end{aligned}
$$

obtaining that

$$
\frac{1}{T} \sum_{|j| \leq n}\left|f\left(e^{j / T}\right)\right|^{2} e^{2 c j / T} \leq\|f\|_{X_{c}^{2}}^{2} .
$$

Using now Hölder's inequality, we have

$$
\begin{aligned}
& \sum_{k=-\infty}^{\infty}\left|\operatorname{lin}_{c / T}\left(e^{-k}(\cdot)^{T}\right) f\left(e^{k / T}\right)\right| \\
\leq & \left(\sum_{k=-\infty}^{\infty}\left|f\left(e^{k / T}\right) e^{c k / T}\right|^{2}\right)^{1 / 2}\left(\sum_{k=-\infty}^{\infty}\left|\operatorname{lin}_{c / T}\left(e^{-k} x^{T}\right) e^{-c k / T}\right|^{2}\right)^{1 / 2} .
\end{aligned}
$$

Now, the first series is convergent, while for the second series we have

$$
\sum_{k=-\infty}^{\infty}\left|\operatorname{lin}_{c / T}\left(e^{-k} x^{T}\right) e^{-c k / T}\right|^{2}=x^{-2 c} \sum_{k=-\infty}^{\infty} \mid \operatorname{sinc}\left(\left.\log \left(e^{-k} x^{T}\right)\right|^{2}\right.
$$

Moreover, using the elementary estimate

$$
|\operatorname{sinc} x| \leq \frac{2}{1+\pi|x|} \quad\left(x \in \mathbb{R}^{+}\right),
$$

we get

$$
\begin{aligned}
& \sum_{k=-\infty}^{\infty}\left|\operatorname{lin}_{c / T}\left(e^{-k} x^{T}\right) e^{-c k / T}\right|^{2} \leq x^{-2 c} \sum_{k=-\infty}^{\infty} \frac{4}{(1+\pi|T \log x-k|)^{2}} \\
\leq & 4 x^{-2 c}\left(2+2 \sum_{k=1}^{\infty} \frac{1}{(1+\pi k)^{2}}\right),
\end{aligned}
$$

where the last inequality is obtained by considering the integral parts of $\mid T \log x-$ $k \mid$, for any $k \in \mathbb{Z}$ and $x>0$. Hence the ESF series is absolutely convergent, thus the exponential sampling series is also absolutely convergent, and moreover the series

$$
x^{c} \sum_{k=-\infty}^{+\infty} f\left(e^{k / T}\right) \operatorname{lin}_{c / T}\left(e^{-k} x^{T}\right)
$$


is uniformly convergent on $\mathbb{R}^{+}$.

In order to justify the interchange of integration and summation, we rewrite the MRKF as follows, using Lemmas 6 and 7,

$$
f(x)=T \int_{0}^{+\infty} f(y) y^{c}\left[y^{-c} \sum_{k=-\infty}^{+\infty} \operatorname{lin}_{c / T}\left(e^{k} y^{-T}\right) \operatorname{lin}_{c / T}\left(e^{-k} x^{T}\right)\right] \frac{d y}{y} .
$$

Observe that the function $F(y):=f(y) y^{c}$ belongs to $L_{\mu}^{2}\left(\mathbb{R}^{+}\right)$. Thus we prove that the sequence

$$
G_{n}(y)=y^{-c} \sum_{|k| \leq n} \operatorname{lin}_{c / T}\left(e^{k} y^{-T}\right) \operatorname{lin}_{c / T}\left(e^{-k} x^{T}\right),
$$

is convergent in $L_{\mu}^{2}\left(\mathbb{R}^{+}\right)$as a function of $y$, obtaining as a consequence of Hölder's inequality the assertion. Using the previous calculations, we have

$$
\begin{aligned}
& \left\|(\cdot)^{-c} \sum_{m \leq|k| \leq n} \operatorname{lin}_{c / T}\left(e^{k}(\cdot)^{-T}\right) \operatorname{lin}_{c / T}\left(e^{-k} x^{T}\right)\right\|_{L_{\mu}^{2}}^{2} \\
= & \sum_{m \leq|k| \leq n} \operatorname{lin}_{c / T}\left(e^{-k} x^{T}\right) \sum_{m \leq|j| \leq n} \operatorname{lin}_{c / T}\left(e^{-j} x^{T}\right)\left\langle(\cdot)^{-c} \operatorname{lin}_{c / T}\left(e^{k}(\cdot)^{-T}\right),(\cdot)^{-c} \operatorname{lin}_{c / T}\left(e^{j}(\cdot)^{-T}\right)\right\rangle_{2} .
\end{aligned}
$$

Now using an elementary subsitution, the scalar product on the right-hand side of the foregoing relation can be written as a scalar product in $X_{c}^{2}$

$$
\left\langle\operatorname{lin}_{c / T}\left(e^{k}(\cdot)^{T}\right), \operatorname{lin}_{c / T}\left(e^{j}(\cdot)^{-T}\right)\right\rangle
$$

Thus, using the orthogonality property (7) of the $\operatorname{lin}_{c}$ functions, we have finally

$$
\begin{aligned}
& \left\|(\cdot)^{-c} \sum_{m \leq|k| \leq n} \operatorname{lin}_{c / T}\left(e^{k}(\cdot)^{-T}\right) \operatorname{lin}_{c / T}\left(e^{-k} x^{T}\right)\right\|_{L_{\mu}^{2}}^{2} \\
= & \frac{1}{T} \sum_{m \leq|k| \leq n}\left|\operatorname{lin}_{c / T}\left(e^{-k} x^{T}\right)\right|^{2} e^{2 c k / T}=\frac{x^{-2 c}}{T} \sum_{m \leq|k| \leq n}\left|\operatorname{sinc}^{2}(T \log x-k)\right| .
\end{aligned}
$$

Since, for every $x \in \mathbb{R}$, by (8) we have

$$
\sum_{k=-\infty}^{\infty}|\operatorname{sinc}(T \log x-k)|^{2}<+\infty
$$

it follows that $\left(G_{n}\right)_{n \in \mathbb{N}}$ is a Cauchy sequence in $L_{\mu}^{2}\left(\mathbb{R}^{+}\right)$, meaning that it converges in $L_{\mu}^{2}\left(\mathbb{R}^{+}\right)$. This means that that the sum in Lemma 7 converges also in $L_{\mu}^{2}$-norm to $G_{x}(\cdot)$. Thus the assertion follows applying Lemma 5. 


\section{Some examples and graphic representations}

In this section we will describe some particular examples of Mellin band-limited and non-bandlimited functions, along with some graphic representation. Firstly, note that the class of Mellin band-limited functions is non-empty and an integral representation of such functions is given in Corollary 4.2 on [13]. In the following we will denote by $\left(S_{N} f\right)(x)$ the partial sum of the exponential sampling series, i.e.

$$
\left(S_{N} f\right)(x)=\sum_{|k| \leq N} f\left(e^{k / T}\right) \operatorname{lin}_{c / T}\left(e^{-k} x^{T}\right)
$$

(a) Let us consider the Mellin-Fejer Kernel defined for $\rho>0$ and $c \in \mathbb{R}$, by

$$
F_{\rho}^{c}(x):=-\frac{x^{-c}}{2 \pi \rho}\left(\frac{x^{\rho i / 2}-x^{-\rho i / 2}}{\log x}\right)^{2}, x \in \mathbb{R}^{+} \backslash\{1\},
$$

with continuous extension $F_{\rho}^{c}(1):=\rho /(2 \pi)$. Its Mellin transform is given by

$$
\left[F_{\rho}^{c}\right]_{M}^{\wedge}(c+i t)=(1-|t| / \rho) \chi_{[-\rho, \rho]}(t),
$$

where $\chi_{A}$ denotes, as usual, the characteristic function of the set $A$. Thus $F_{\rho}^{c}$ is Mellin band-limited. So, taking into account that now $T=\rho / \pi$, the ESF takes on for (9) the concrete form

$$
\begin{aligned}
F_{\rho}^{c}(x) & =\sum_{k=-\infty}^{\infty} F_{\rho}^{c}\left(e^{k \pi / \rho}\right) \operatorname{lin}_{c \pi / \rho}\left(e^{-k} x^{\rho / \pi}\right) \\
& =\frac{2 \rho}{\pi^{3} x^{c}} \sum_{k=-\infty}^{\infty} \frac{\sin ^{2}(k \pi / 2)}{k^{2}} \operatorname{sinc}\left(\frac{\rho}{\pi} \log x-k\right) .
\end{aligned}
$$

In practice one cannot deal with an infinite series but only with a finite sum. This leads to an additional error, namely the truncation error

$$
\left(T_{\rho, N} F_{\rho}^{c}\right)(x)=\frac{2 \rho}{\pi^{2} x^{c}} \sum_{|k| \geq N+1} \frac{\sin ^{2}(k \pi / 2)}{k^{2}} \operatorname{sinc}\left(\frac{\rho}{\pi} \log x-k\right) .
$$

In order to obtain an estimate for this error which is independent of $x$, we can choose $N$ depending on $x$. Putting, for example

$$
N \geq 2 \max \left\{x^{-c}, \frac{\rho}{\pi}|\log x|\right\}
$$

we have

$$
\left|\operatorname{sinc}\left(\frac{\rho}{\pi} \log x-k\right)\right| \leq \frac{1}{\pi(|k|-|(\rho / \pi) \log x|} \leq \frac{2}{\pi|k|} \quad(|k| \geq N+1) .
$$


This implies for the truncation error

$$
\begin{aligned}
\left|\left(T_{\rho, N} F_{\rho}^{c}\right)(x)\right| & \leq \frac{4 \rho}{\pi^{4} x^{c}} \sum_{|k| \geq N+1} \frac{1}{|k|^{3}} \leq \frac{4 \rho}{\pi^{4} x^{c}} \int_{N}^{\infty} \frac{d u}{u^{3}} \\
& \leq \frac{4 \rho}{\pi^{4} x^{c}} N^{-2} \leq \frac{4 \rho}{\pi^{4}} N^{-1}
\end{aligned}
$$

Thus one has

$\left|F_{\rho}^{c}(x)-\left(S_{N} F_{\rho}^{c}\right)(x)\right| \leq\left|\left(T_{\rho, N} F_{\rho}^{c}\right)(x)\right| \leq \frac{4 \rho}{\pi^{4}} N^{-1} \quad\left(N \geq 2 \max \left\{x^{-c}, \frac{\rho}{\pi}|\log x|\right)\right.$.

$\left(S_{N} F_{\rho}^{c}\right)(x)$ being the $N$-th partial sum of (10).

Note that this method also gives a better order of pointwise approximation.

As a particular example, let us consider the Mellin-Fejer kernel $F_{\pi}^{1}(x)$, corresponding to the case $c=1, \rho=\pi$ (see Fig.1). In this case, we have $T=1$, and the ESF formula takes on the form

$$
F_{\pi}^{1}(x)=\lim _{N \rightarrow+\infty}\left(S_{N} F_{\pi}^{1}\right)(x)
$$

Below in Fig.1 are the graphs of $\left(S_{1} F_{\pi}^{1}\right),\left(S_{3} F_{\pi}^{1}\right)$ and $\left(S_{5} F_{\pi}^{1}\right)$ showing their approximations to the function $F_{\pi}^{1}$.

(b) Here we consider an important example of non-bandlimited functions, namely the Mellin-Abel-Poisson kernel (see [12]) defined, for $\rho>0$, by

$$
P_{\rho}(x)= \begin{cases}\frac{\rho}{2} x^{\rho}, & 0<x \leq 1 \\ \frac{\rho}{2} x^{-\rho}, & x>1\end{cases}
$$

The Mellin transform of $P_{\rho}$ can be evaluated on every line $c+i t$, with $c \in]-\rho, \rho[$ and $t \in \mathbb{R}$. For example, for $c=0$ it is given by

$$
\left[P_{\rho}\right]_{M}^{\wedge}(i t)=\left[1-\left(\frac{i t}{\rho}\right)^{2}\right]^{-1}=\frac{\rho^{2}}{\rho^{2}+t^{2}}
$$

which is integrable as a function of $t$ over the real line. Thus the function $P_{\rho}$ satisfies all the assumptions of Theorem 3 .

The partial sums of the exponential sampling series, with $c=0$, are now given, for $N \in \mathbb{N}$, by

$$
\left(S_{N} P_{\rho}\right)(x)=\frac{\rho}{2} \sum_{|k| \leq N} \exp (-\rho|k / T|) \operatorname{sinc}(T \log x-k) .
$$



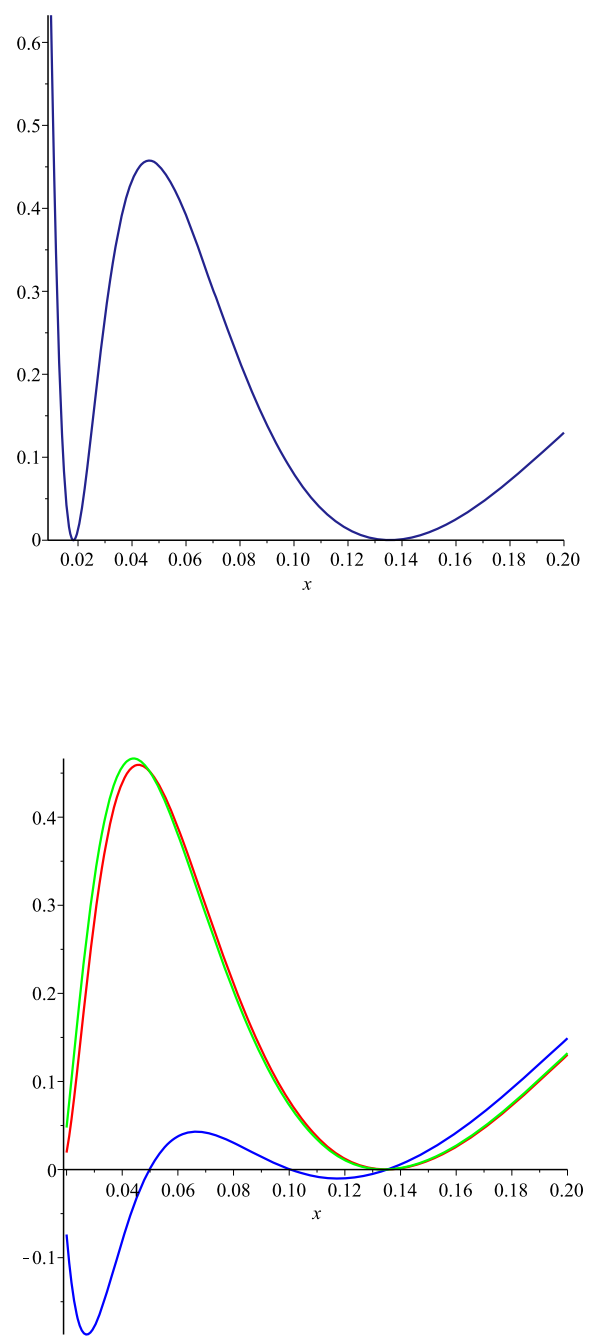

Figure 1: The function $F_{\pi}^{1}$ (blue) at the left and the partial sums $\left(S_{1} F_{\pi}^{1}\right)$ (navy), $\left(S_{3} F_{\pi}^{1}\right)$ (green) and $\left(S_{5} F_{\pi}^{1}\right)$ (red) at the right, in the interval $[0.02,0.20]$ 
In order to estimate the error of approximation of $P_{\rho}(x)$ by the partial sums $\left(S_{N} P_{\rho}\right)(x)$, the truncation error, we use now the approximate exponential sampling theorem (Theorem 3). For $c=0$ and $T>0$, choosing now $N \geq 2|T \log x|$, as in Example (a), we have

$$
|\operatorname{sinc}(T \log x-k)| \leq \frac{2}{\pi|k|} \quad(|k| \geq N+1),
$$

so that

$$
\begin{aligned}
\left|P_{\rho}(x)-\left(S_{N} P_{\rho}\right)(x)\right| & \leq \frac{\rho}{2} \sum_{|k| \geq N+1} \exp (-\rho|k / T|)|\operatorname{sinc}(T \log x-k)| \\
& +\frac{1}{\pi} \int_{|t|>\pi T}\left|\left[P_{\rho}\right]_{M}^{\wedge}(i t)\right| d t \\
& \leq \frac{\rho}{\pi} \sum_{|k| \geq N+1} \frac{e^{-\rho|k| / T}}{|k|}+\frac{\rho}{\pi} \int_{|u|>\pi T / \rho} \frac{1}{1+u^{2}} d u=: I_{1}+I_{2} .
\end{aligned}
$$

Now

$$
I_{1}=\frac{\rho}{\pi} \sum_{|k| \geq N+1} \frac{e^{-\rho|k| / T}}{|k|} \leq \frac{\rho}{\pi} \int_{\rho N / T} \frac{e^{-v}}{v} d v .
$$

The last integral is the well-known Exponential Integral

$$
E_{1}(x):=\int_{x}^{\infty} \frac{e^{-u}}{u} d u \quad(x>0)
$$

which can be estimated by (see e.g. [1], page 229)

$$
E_{1}(x) \leq \frac{e^{-x}}{x} \quad(x>0)
$$

Thus we have, for the truncation error,

$$
I_{1} \leq \frac{T}{\pi N} e^{-\rho N / T}, \quad(N \geq 2|T \log x|) .
$$

For $I_{2}$ we get

$$
I_{2}=\frac{\rho}{\pi} \int_{|u|>\pi T / \rho} \frac{1}{1+u^{2}} d u \leq \frac{2 \rho}{\pi} \int_{\pi T / \rho}^{\infty} \frac{1}{u^{2}} d u=2 \frac{\rho^{2}}{\pi^{2}} T^{-1} .
$$

Finally, for any fixed $x>0$, we obtain the error estimate

$$
\left|P_{\rho}(x)-\left(S_{N} P_{\rho}\right)(x)\right| \leq \frac{T}{\pi N} e^{-\rho N / T}+2 \frac{\rho^{2}}{\pi^{2}} T^{-1}, \quad(N \geq 2|T \log x|)
$$


which tends to zero for $T$ tending to $\infty$, the limit of the first term depending on the choice of $N$ and $T$ (valid in particular for $N \geq T^{\gamma}$, any $\gamma>1$ ), and the aliasing error is then of the order $\mathcal{O}\left(T^{-1}\right)$.

Now we consider the case $c \in] \rho, \rho[\backslash\{0\}$. Let us put $a:=c+\rho>0$ and $b:=\rho-c>0$. In this instance the partial sums take the form

$$
\begin{aligned}
\left(S_{N} P_{\rho}\right)(x) & =\frac{\rho}{2} \sum_{|k| \leq N} \exp (-\rho|k| / T) \operatorname{lin}_{c / T}\left(e^{-k} x^{T}\right) \\
& =\frac{\rho x^{-c}}{2} \sum_{|k| \leq N} \exp \left(\frac{1}{T}(-\rho|k|+c k)\right) \operatorname{sinc}(T \log x-k) .
\end{aligned}
$$

Moreover the Mellin transform on the line $s=c+i t$ is given by

$$
\left[P_{\rho}\right] \wedge(c+i t)=\frac{\rho^{2}}{\rho^{2}-s^{2}} \quad(s=c+i t ; t \in \mathbb{R}),
$$

so that $\left[P_{\rho}\right]_{M}^{\wedge} \in L^{1}(\{c\} \times \mathbb{R})$ as a function of $t \in \mathbb{R}$. As in Example (a), let us take $N \geq 2 \max \left\{x^{-c},|T \log x|\right\}$, so that

$$
\mid \operatorname{sinc}(T \log x-k \mid) \leq \frac{2}{\pi T}, \quad(|k| \geq N+1) .
$$

Then

$$
\begin{aligned}
\left|P_{\rho}(x)-\left(S_{N} P_{\rho}\right)(x)\right| & \leq \frac{\rho x^{-c}}{\pi} \sum_{|k| \geq N+1} \frac{\exp ((-\rho|k|+c k) / T)}{|k|} \\
& +\frac{x^{-c}}{\pi} \int_{|t|>\pi T}\left|\left[P_{\rho}\right]_{M}^{\wedge}(c+i t)\right| d t:=J_{1}+J_{2} .
\end{aligned}
$$

For $J_{1}$ putting $r:=\min \{a, b\}$, we can write

$$
J_{1} \leq \frac{\rho x^{-c}}{\pi} \sum_{k=-\infty}^{-(N+1)} \frac{e^{a k / T}}{|k|}+\frac{\rho x^{-c}}{\pi} \sum_{k=N+1}^{\infty} \frac{e^{-b k / T}}{k} \leq \frac{2 \rho x^{-c}}{\pi} \sum_{k=N+1}^{\infty} \frac{e^{-r k / T}}{k} .
$$

Therefore, proceeding as before, using the Exponential Integral, we have

$$
J_{1} \leq \frac{\rho T}{\pi} e^{-r N / T}, \quad\left(N \geq 2 \max \left\{x^{-c},|T \log x|\right\}\right) .
$$

As to $J_{2}$, we have

$$
J_{2} \leq \frac{x^{-c}}{\pi} \int_{|t|>\pi T} \frac{\rho^{2}}{t^{2}} d t \leq \frac{2 x^{-c} \rho^{2}}{\pi^{2}} T^{-1}
$$


Thus for every fixed $x>0$,

$\left|P_{\rho}(x)-\left(S_{N} P_{\rho}\right)(x)\right| \leq \frac{\rho T}{2 \pi} e^{-r N / T}+\frac{2 x^{-c} \rho^{2}}{\pi^{2}} T^{-1}, \quad\left(N \geq 2 \max \left\{x^{-c},|T \log x|\right\}\right)$,

which tends to zero, if we choose $N, T$ such that $N / T \rightarrow+\infty$ for $T \rightarrow+\infty$. The truncation error is independent of $x$ while the aliasing error depends on $x$. If we choose also the parameter $T$ depending on $x$, for example $T^{1-\alpha}>x^{-c}$, where $\left.\alpha \in\right] 0,1\left[\right.$, then the aliasing error, namely $2 \rho^{2} / \pi^{2} T^{\alpha}$, is independent of $x$, but tends to zero slowly for $T \rightarrow+\infty$.

In Fig. 2, in case $c=0$, are the graphs of $P_{1 / 2}$ near the point 1, along with the partial sums $S_{N} P_{1 / 2}$ with $T=4$, and for $N=6,8,10$, and the graphs of the same functions but in the very critical interval ]0,1/3[, (especially at $x=0$ ), with $N=10,12,14$. Finally, in Fig. 3 we have plotted the graphs of the function $P_{1}$ and the respective partial sums with the same values of $T$ and $N$, near to the point 1 and to the point 0 .

(c) Here we consider another example of a non-bandlimited signal, belonging to the space $X_{1}=L^{1}\left(\mathbb{R}^{+}\right)$. We define (see Fig. 3 )

$$
g(x)=\log x \chi_{] 0,1[}(x) .
$$

Its Mellin transform on the line $s=1+i t, t \in \mathbb{R}$, is given by

$$
[g]_{M}^{\wedge}(1+i t)=\frac{1}{(1+i t)^{2}},
$$

and so it is integrable on $\{1\} \times \mathbb{R}$. Hence, the assumptions of Theorem 3 are satisfied. The corresponding exponential series is given, for $T>0$, by

$$
\frac{1}{T x} \sum_{k=-\infty}^{-1} k e^{k / T} \operatorname{sinc}(T \log x-k),
$$

and its partial sums are

$$
\left(S_{N} g\right)(x)=\frac{1}{T x} \sum_{k=-N}^{-1} k e^{k / T} \operatorname{sinc}(T \log x-k) .
$$

For a fixed $x>0$ as in Example (a) we choose $N \geq 2 \max \left\{x^{-1},|T \log x|\right\}$, obtaining

$$
\begin{aligned}
& \left|g(x)-\left(S_{N} g\right)(x)\right| \leq \frac{2}{T x} \sum_{k=-\infty}^{-(N+1)} e^{k / T}+\frac{1}{\pi x} \int_{|t|>\pi T}\left|[g]_{M}^{\wedge}(1+i t)\right| d t \\
& =: H_{1}+H_{2} .
\end{aligned}
$$



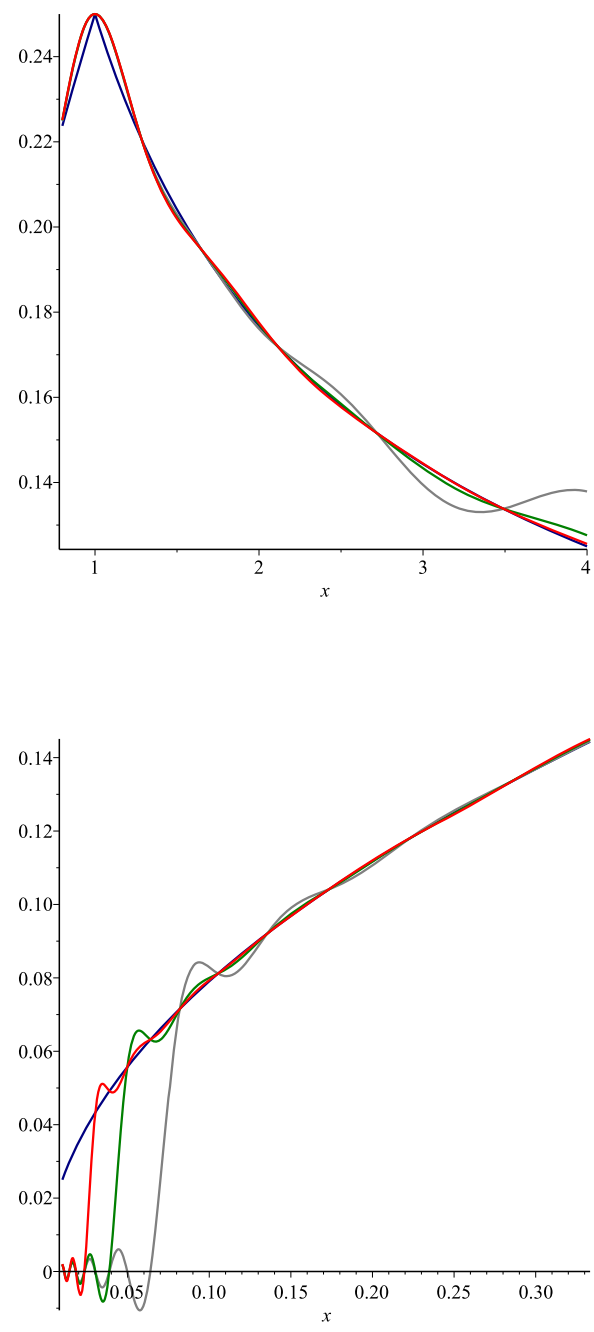

Figure 2: The function $P_{1 / 2}$ (navy) $\left(S_{6} P_{1 / 2}\right)$ (gray), $\left(S_{8} P_{1 / 2}\right)$ (green) and $\left(S_{10} P_{1 / 2}\right)$ (red) near 1 on the left; The function $P_{1 / 2}$ (navy) $\left(S_{10} P_{1 / 2}\right)$ (gray), $\left(S_{12} P_{1 / 2}\right)$ (green) and $\left(S_{14} P_{1 / 2}\right)$ (red) near 0 on the right 

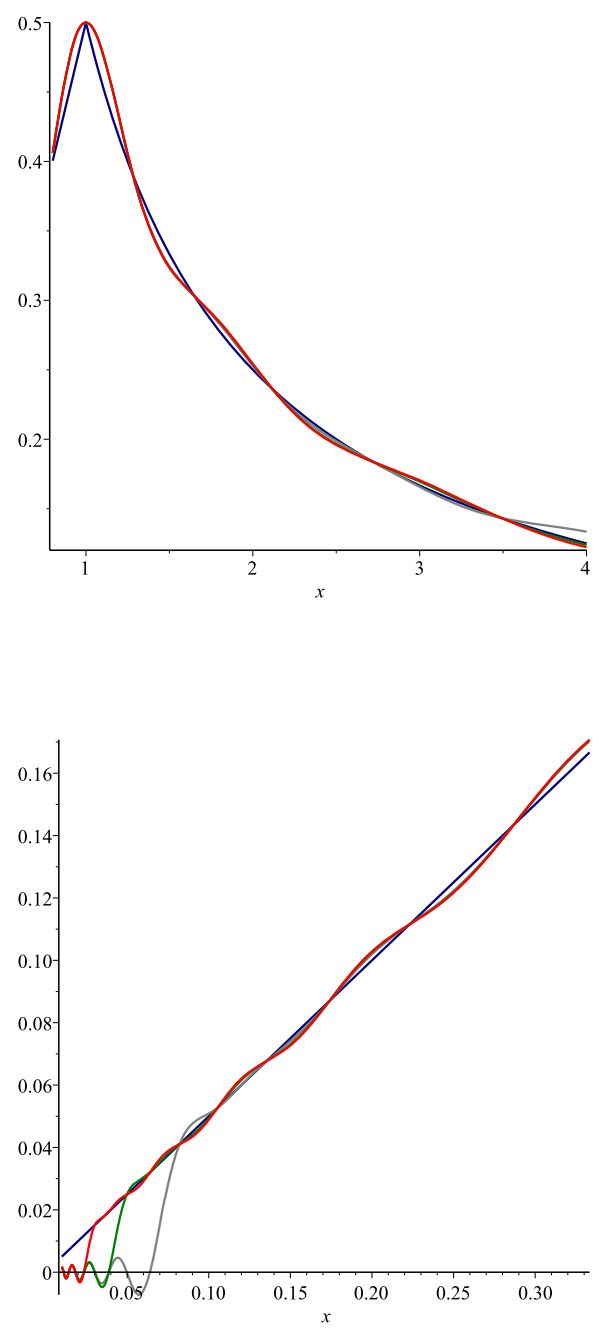

Figure 3: The function $P_{1}$ (blue) and $\left(S_{6} P_{1}\right)$ (gray), $\left(S_{8} P_{1}\right)$ (green) and $\left(S_{10} P_{1}\right)$ (red) near the point 1 on the left; The function $P_{1}$ (blue) and $\left(S_{10} P_{1}\right)$ (gray), $\left(S_{12} P_{1}\right)$ (green) and $\left(S_{14} P_{1}\right)$ (red) near the point 0 on the right 
For $H_{1}$ we have, by calculating the sum of the series and using the fact that $t /\left(e^{t}-1\right) \leq 1$ for every $t>0$,

$$
H_{1} \leq \frac{2}{T x} \frac{e^{-N / T}}{e^{1 / T}-1} \leq N e^{-N / T} \quad\left(N \geq 2 \max \left\{x^{-1},|T \log x|\right\}\right) .
$$

For $\mathrm{H}_{2}$ we have

$$
H_{2} \leq \frac{2}{\pi^{2} x} T^{-1},
$$

Therefore we obtain the estimate

$$
\left|g(x)-\left(S_{N} g\right)(x)\right| \leq N e^{-N / T}+\frac{2}{\pi^{2} x} T^{-1} \quad\left(N \geq 2 \max \left\{x^{-1},|T \log x|\right\}\right),
$$

which tends to zero if we choose $N, T$ such that $N e^{-N / T}$ tends to zero as $T \rightarrow+\infty$, (we can choose again $N \geq T^{\gamma}$, any $\gamma>1$ ). Also in this case the aliasing error depends on $x$. If for any fixed $x>0$, we choose $T$ such that again $T^{1-\alpha} \geq x^{-1}$, then we obtain an error estimate which is independent of $x$.

Below in Fig.4 are the graphs of $\left(S_{4} g\right),\left(S_{6} g\right)$ and $\left(S_{8} g\right)$ for $T=3$ all plotted together; they approximate the function $g$.

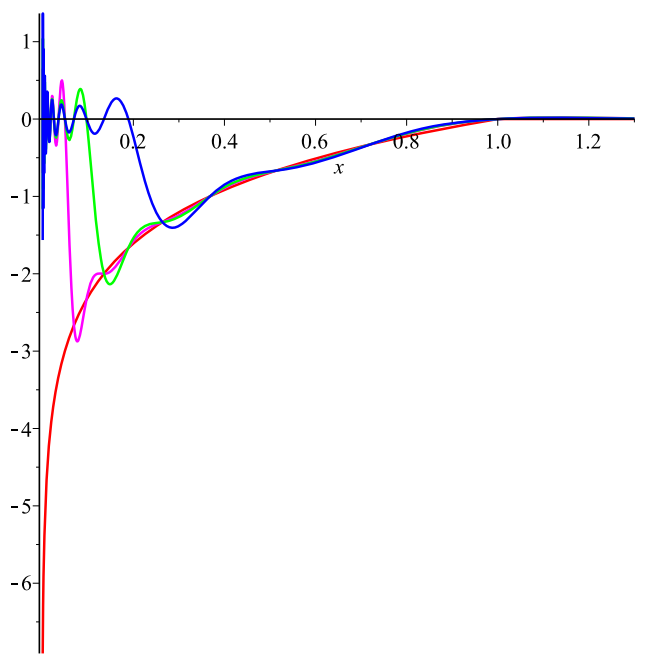

Figure 4: The functions $g$ (red) and $\left(S_{4} g\right)$ (blue), $\left(S_{6} g\right)$ (green) and $\left(S_{8} g\right)$ (magenta) for $T=3$ in the interval $[0.02,1.3]$ 


\section{ACKNOWLEDGEMENT}

We wish to thank Professor Rudolf Stens for his suggestions concerning the independency of the truncation error upon $x$ in example (a), and Professor Gianluca Vinti for providing us with the many graphs of Section 6, using Maple Package. Finally we wish to thank the Referee for his constructive report.

The first and third authors have been partially supported by the Gruppo Nazionale Analisi Matematica, Probabilitá e Applicazioni (GNAMPA) of the Istituto Nazionale di Alta Matematica (INdAM) and by the Department of Mathematics and Computer Sciences of University of Perugia.

\section{References}

[1] M. Abramowitz and I.A. Stegun (Eds), Handbook of mathematical functions, Nstional Bureau of Standars. USA, Applied Math. Series 55, X Edition, 1972.

[2] M.H. Annaby and P.L. Butzer, Mellin type differential equations and associated sampling expansions, Numer. Funct. Anal. Optim., 21, 1-24 (2000).

[3] C. Bardaro, P.L. Butzer and I. Mantellini, Mellin Analysis and Exponential Sampling. Part I: Mellin fractional integrals, in Proceedings of 10th International Conference on Sampling Theory and Applications, Eurasip Open Library, 274-276, (2013).

[4] C. Bardaro, P.L. Butzer and I. Mantellini, Mellin Analysis and Exponential Sampling. Part II: Mellin differential operaators and sampling, in Proceedings of 10th International Conference on Sampling Theory and Applications, Eurasip Open Library, 277-280, (2013).

[5] C. Bardaro, P.L. Butzer and I. Mantellini, The foundations of fractional Mellin transform analysis, to appear.

[6] C. Bardaro, P.L. Butzer, R. Stens and G. Vinti, Approximation error of the Whittaker cardinal series in terms of an averaged modulus of smoothness covering discontinuous signals, J. Math. Anal. Appl.,316, 269-306, (2006).

[7] P.T. Bateman and H.G. Diamond, Analytic number theory. An introductory course, World Scientific, Singapore, 2004.

[8] M. Bertero and E.R. Pike, Exponential sampling method for Laplace and other dilationally invariant transforms I. Singular-system analysis. II. Examples in photon correction spectroscopy and Frauenhofer diffraction,Inverse Problems, 7, 1-20; 21-41, (1991). 
[9] P.L. Butzer, P.J.S.G. Ferreira, R.J.Higgins, G.Schmeisser, R.L. Stens, The sampling theorem, Poisson's summation formula, general Parseval formula, reproducing kernel formula and the Paley-Wiener theorem for bandlimited signals-their interconnections, Appl. Anal., 90 (3-4), 431-461, (2011).

[10] P.L. Butzer, M. Dodson, P.J.S.G. Ferreira, R.J.Higgins, G.Schmeisser and R.L. Stens, The generalized Parseval decomposition formula, the approximate sampling theorem, the approximate reproducing kernel formula, Poisson's summation formula, and Riemann's Zeta function; their interconnections for nonbandilimited functions, to appear.

[11] P.L. Butzer and S. Jansche, Mellin transform theory and the role of its differential and integral operators, Proc. Second Int. Workshop "Transform methods and special functions", Varna, 63-83, (1996).

[12] P.L. Butzer and S. Jansche, A direct approach to the Mellin transform, $J$. Fourier Anal. Appl., 3, 325-375, (1997).

[13] P.L. Butzer and S. Jansche, The exponential sampling theorem of signal analysis, Atti Sem. Mat. Fis. Univ. Modena, Suppl. Vol.46, 99-122, (1998), special issue dedicated to Professor Calogero Vinti.

[14] P.L. Butzer and S. Jansche, A self-contained approach to Mellin transform analysis for square integrable functions; applications, Integral Transforms and Special Functions, 8(3-4), 175-198, (1999).

[15] P.L. Butzer, A.A. Kilbas and J.J. Trujillo, Mellin transform analysis and integration by parts for Hadamard-type fractional integrals, J. Mat. Anal. Appl., 270, 1-15, (2002).

[16] P.L. Butzer, W. Splettstößer and R.L. Stens, The sampling theorem and linear prediction in signal analysis, Jahresber. Deutsch. Math.-Verein.,90, 1-70, (1988).

[17] D. Casasent (Ed), Optical data processing, Springer, Berlin, 241-282, (1978).

[18] F. Gori, Sampling in optics, in Advances Topics in Shannon Sampling and Interpolation Theory (R.J. Marks II Ed), Springer, New York, 37-83, (1993).

[19] J. R. Higgins, Sampling theory in Fourier and signal analysis, Foundations, Oxford Univ. Press, Oxford, 1996.

[20] N. Ostrowsky, D. Sornette, P. Parker and E.R. Pike, Exponential sampling method for light scattering polydispersity analysis, Opt. Acta, 28, 10591070, (1994). 
[21] R.G. Mamedov, The Mellin transform and approximation theory, (in Russian), "Elm", Baku, 1991.

[22] S.J. Patterson,An introduction to the Riemann Zeta function, Cambridge Studies Advanced Math., 14, Cambridge Univ. Press, Cambridge, 1988.

[23] S.G. Samko, A.A. Kilbas and O.I. Marichev, Fractional Integrals and Derivatives. Theory and Applications, Yverdon: Gordon and Breach, Amsterdam, 1993.

[24] E.C. Titchmarsh, Introduction to the theory of Fourier integrals. Third Edition., Chelsea Publ. co., New York, 1986.

[25] A. I. Zayed, Advances in Shannon's sampling theory, CRC Press, Boca Raton, 1993. 